\title{
INFRARED OBSERVATIONS OF QSS
}

\author{
F. J. Low \\ (Lunar and Planetary Laboratory, University of Arizona, Tucson, Ariz., U.S.A.)
}

No abstract has been submitted. 